\title{
Article \\ Implicit Hybrid Fractional Boundary Value Problem via Generalized Hilfer Derivative
}

\author{
Abdellatif Boutiara ${ }^{1,+}\left(\mathbb{D}\right.$, Mohammed S. Abdo ${ }^{2,+}$, Mohammed A. Almalahi ${ }^{3,+} \mathbb{D}$, Hijaz Ahmad $^{4, *,+, \mathbb{D}}$ \\ and Amira Ishan 5 \\ 1 Laboratory of Mathematics and Applied Sciences, University of Ghardaia, Metlili 47000, Algeria; \\ boutiara_a@yahoo.com \\ 2 Department of Mathematics, College of Education, Hodeidah University, P.O. Box 3114, Al-Hudaydah 207416, \\ Yemen; msabdo@hoduniv.net.ye \\ 3 Department of Mathematics, Hajjah University, Hajjah 00967, Yemen; dralmalahi@gmail.com \\ 4 Section of Mathematics, International Telematic University Uninettuno, Corso Vittorio Emanuele II, 39, \\ 00186 Roma, Italy \\ 5 Department of Mathematics, College of Science, Taif University, P.O. Box 11099, Taif 21944, Saudi Arabia; \\ a.ishan@tu.edu.sa \\ * Correspondence: hijaz555@gmail.com \\ $+\quad$ These authors contributed equally to this work.
}

check for updates

Citation: Boutiara, A.; Abdo, M.S.; Almalahi, M.; Ahmad, H.; Ishan A Implicit Hybrid Fractional Boundary Value Problem via Generalized Hilfer Derivative. Symmetry 2021, 13, 1937. https://doi.org/10.3390/ sym13101937

Academic Editor: Calogero Vetro

Received: 05 September 2021

Accepted: 11 October 2021

Published: 15 October 2021

Publisher's Note: MDPI stays neutral with regard to jurisdictional claims in published maps and institutional affiliations.

Copyright: (c) 2021 by the authors. Licensee MDPI, Basel, Switzerland. This article is an open access article distributed under the terms and conditions of the Creative Commons Attribution (CC BY) license (https:/ / creativecommons.org/licenses/by/ $4.0 /)$.

\begin{abstract}
This research paper is dedicated to the study of a class of boundary value problems for a nonlinear, implicit, hybrid, fractional, differential equation, supplemented with boundary conditions involving general fractional derivatives, known as the $\vartheta$-Hilfer and $\vartheta$-Riemann-Liouville fractional operators. The existence of solutions to the mentioned problem is obtained by some auxiliary conditions and applied Dhage's fixed point theorem on Banach algebras. The considered problem covers some symmetry cases, with respect to a $\vartheta$ function. Moreover, we present a pertinent example to corroborate the reported results.
\end{abstract}

Keywords: $\vartheta$-Hilfer fractional operator; implicit; Dhage fixed point theorem; Banach algebras

\section{Introduction}

Fractional calculus theory is an extension and expansion of integer calculus theory. Because of its good heritability and memory, it has received wide attention from more and more scholars. It is used in optical and thermal systems, rheology and material mechanics systems, signal processing and system identification, as well as control and robotics and has a wide range of applications [1-5]. Fractional theory concepts are used in modern studies to describe the state of complex physical systems with self-similarity properties, complex scaling, and heredity. The methods of fractal and multifractal analysis of images and time series are presented as mathematical tools, allowing the specification of irregular objects and dynamic properties of processes. As a fractal formalism (or independent scientific theory), fractional calculus is one of the recent analytical directions. The most used fractional operators are the Riemann-Liouville and Caputo types [1,2]. Recently, many generalizations of these fractional operators have appeared, one such general category is the class of fractional operators involving analytic kernels, introduced by Fernandez et al. [6]. Another, more broad, class of fractional operators, sometimes referred to as $\psi$-fractional calculus, is described by fractional differentiation and the integration of one function, regarding another function. These generalizations are a useful method for generalizing many kinds of fractional calculus, which have been intensely considered over the most recent few years. We mention here a simple survey within this class, which will be a significant subject of this paper, namely developed fractional calculus, which has likewise been called $\psi$-fractional calculus. The thought previously emerged in a 1964 research paper by Erdelyi [7], in which he examined the fractional calculus regarding a power function $x^{n}$; 
a similar operator was proposed by Katugampola in 2011 [8]. Generality, this category was first proposed and inspired by Osler in 1970 [9], and it was mentioned briefly in the 1974 textbook [10]; it was then taken up extensively in the monograph of Kilbas et al. 2006 [1]. As of late, Almeida [11] and Sousa et al. [12] have used fractional operators to generalize Caputo and Hilfer types, respectively, with respect to another function. Additionally, Jarad and Thabet, in [13], presented fascinating properties of this generalized operator, including the generalized Laplace transform.

On the other hand, there has been a great deal of interest in the field of the qualitative analysis of fractional differential equations (FDEs), with multiple boundary conditions involving the generalized Hilfer operator. For more details, see [14-18]. For the recent works regarding to proposed problem, see [19-24].

Wang and Zhang [22] investigated some existence results of the following IVP, for a nonlocal FDE under the Hilfer derivative:

$$
\left\{\begin{array}{l}
\mathcal{D}_{a^{+}}^{Q, \beta} u(\varkappa)=\mathcal{F}(\varkappa, u(\varkappa)), \quad \varkappa \in(a, b], \\
\mathcal{I}_{a^{+}}^{1-\gamma ; \vartheta} u(a)=\sum_{j=1}^{m} c_{j} u\left(\tau_{j}\right), \tau_{j} \in(a, b) .
\end{array}\right.
$$

Derbazi et al. [23] proved the existence and uniqueness results of the following BVP, for a hybrid FDE under Caputo derivatives:

$$
\left\{\begin{array}{l}
{ }^{C} \mathcal{D}_{0^{+}}^{\varrho}\left(\frac{u(\varkappa)-\mathcal{F}(\varkappa, u(\varkappa))}{\mathcal{G}(\varkappa, u(\varkappa))}\right)=\mathcal{H}(\varkappa, u(\varkappa)), \quad \varkappa \in[0, T] \\
a_{1}\left(\frac{u(0)-\mathcal{F}(0, u(0))}{\mathcal{G}(0, u(0))}\right)+b_{1}\left(\frac{u(T)-\mathcal{F}(T, u(T))}{\mathcal{G}(T, u(T))}\right)=c_{1} \\
a_{2}{ }^{C} \mathcal{D}_{0^{+}}^{\sigma}\left(\frac{u(\eta)-\mathcal{F}(\eta, u(\eta))}{\mathcal{G}(\eta, u(\eta))}\right)+b_{2}{ }^{C} \mathcal{D}_{0^{+}}^{\sigma}\left(\frac{u(T)-\mathcal{F}(T, u(T))}{\mathcal{G}(T, u(T))}\right)=c_{2}, \quad \eta \in(0, T) .
\end{array}\right.
$$

Salim et al. [24] established the existence results of the following IVP for a nonlocal implicit hybrid FDE under the Katugampola Hilfer derivative:

$$
\left\{\begin{array}{l}
{ }^{\rho} \mathcal{D}_{a^{+}}^{\varrho, \beta}\left(\frac{u(\varkappa)-\mathcal{F}(\varkappa, u(\varkappa))}{\mathcal{G}(\varkappa, u(\varkappa))}\right)=\mathcal{H}\left(\varkappa, u(\varkappa),{ }^{\rho} \mathcal{D}_{a^{+}}^{\varrho, \beta}\left(\frac{u(\varkappa)-\mathcal{F}(\varkappa, u(\varkappa))}{\mathcal{G}(\varkappa, u(\varkappa))}\right)\right), \varkappa \in(a, b], \\
\rho^{\rho} \mathcal{I}_{a^{+}}^{1-\gamma}\left(\frac{u(a)-\mathcal{F}(a, u(a))}{\mathcal{G}(a, u(a))}\right)=\sum_{j=1}^{m} c_{j}\left(\frac{u\left(\tau_{j}\right)-\mathcal{F}\left(\tau_{j}, u\left(\tau_{j}\right)\right)}{\mathcal{G}\left(\tau_{j}, u\left(\tau_{j}\right)\right)}\right) .
\end{array}\right.
$$

Motivated by recent developments in the topic of generalized fractional calculus (and according to the available ideas in the above studies), in the present study, we intend to address a general extension of these works by performing existence analysis on the following BVPs for a nonlinear implicit hybrid FDE with mixed integro-derivative boundary conditions:

$$
\left\{\begin{array}{l}
\mathcal{D}_{a^{+}}^{\varrho, \beta ; \vartheta}\left(\frac{u(\varkappa)-\mathcal{F}(\varkappa, u(\varkappa))}{\mathcal{G}(\varkappa, u(\varkappa))}\right)=\mathcal{H}\left(\varkappa, u(\varkappa), \mathcal{D}_{a^{+}}^{\varrho, \beta ; \vartheta}\left(\frac{u(\varkappa)-\mathcal{F}(\varkappa, u(\varkappa))}{\mathcal{G}(\varkappa, u(\varkappa))}\right)\right), \quad \varkappa \in J:=[a, b], \\
a_{1} \mathcal{I}_{a^{+}}^{1-\gamma ; \vartheta}\left(\frac{u(a)-\mathcal{F}(a, u(a))}{\mathcal{G}(a, u(a))}\right)+b_{1}\left(\frac{u(b)-\mathcal{F}(b, u(b))}{\mathcal{G}(b, u(b))}\right)=c_{1}, \\
a_{2} \mathcal{D}_{a^{+}}^{\sigma, \beta ; \vartheta}\left(\frac{u(\eta)-\mathcal{F}(\eta, u(\eta))}{\mathcal{G}(\eta, u(\eta))}\right)+b_{2} \mathcal{D}_{a^{+}}^{\sigma, \beta ; \vartheta}\left(\frac{u(b)-\mathcal{F}(b, u(b))}{\mathcal{G}(b, u(b))}\right)=c_{2}, \quad \eta \in(a, b),
\end{array}\right.
$$

where $\mathcal{D}_{a^{+}}^{\varrho, \beta ; \vartheta}$ and $\mathcal{D}_{a^{+}}^{\sigma, \beta ; \vartheta}$ are the $\vartheta$-Hilfer fractional derivatives ( $\vartheta$-Hilfer FDs for short) of order $\varrho \in(1,2)$ and $\sigma \in(0,1)$, respectively; type $\beta \in(0,1), \mathcal{I}_{a^{+}}^{1-\gamma ; \vartheta}$ is the $\vartheta$-RiemannLiouville $(\vartheta$-RL for short) fractional integral of order $1-\gamma, \gamma \stackrel{a}{=} \varrho+\beta(2-\varrho)$; and the 
non-linear functions $\mathcal{G}: J \times \mathbb{R} \rightarrow \mathbb{R} \backslash\{0\}, \mathcal{F}: J \times \mathbb{R} \longrightarrow \mathbb{R}$, and $\mathcal{H}: J \times \mathbb{R} \times \mathbb{R} \longrightarrow \mathbb{R}$ are continuous, such that $\mathcal{F}(a, u(a))=0$.

Observe that the problem (4) is new to the literature on implicit hybrid FDEs and includes a hybrid FDE (2) for $a=0, \beta=1$, and $\vartheta(\varkappa)=\varkappa$. Moreover, our current problem generates many problems associated with different values of $\beta$ and $\vartheta$ as special cases. The proposed problem covers some symmetry cases, with respect to the $\vartheta$ function and parameter $\beta$. For instance, in the case of $\vartheta(\varkappa)=\varkappa$ and $\vartheta(\varkappa)=\log \varkappa$, our problem (4) symmetries the Hilfer type hybrid problem and Hilfer-Hadamard type hybrid problem. Besides, if $a_{1}=a_{2}=1, b_{1}=b_{2}=-1$, and $c_{1}=c_{2}=0$, then the conditions of Equation (4) become the symmetry conditions $\left(\frac{u(a)-\mathcal{F}(a, u(a))}{\mathcal{G}(a, u(a))}\right)=\left(\frac{u(b)-\mathcal{F}(b, u(b))}{\mathcal{G}(b, u(b))}\right)$ for the values of $a, b$ with $\gamma=1$ and $\mathcal{D}_{a^{+}}^{\sigma, \beta ; \vartheta}\left(\frac{u(a)-\mathcal{F}(a, u(a))}{\mathcal{G}(a, u(a))}\right)=\mathcal{D}_{a^{+}}^{\sigma, \beta ; \vartheta}\left(\frac{u(b)-\mathcal{F}(b, u(b))}{\mathcal{G}(b, u(b))}\right)$ for the fractional derivative of order $(\sigma, \beta)$ of the unknown function $\frac{u(\cdot)-\mathcal{F}(\cdot, u(\cdot))}{\mathcal{G}(\cdot, u(\cdot))}$.

The structure of our paper is provided as follows. Section 2 contains the definitions and preliminary results to prove our main findings. In Section 3, we present an assistive lemma that extracts the representation for the solutions of the problem (4) and we establish the existence of the solutions by taking advantage of the Dhage fixed point theorem for the proposed problem. The last section gives an illustrative example to support and justify the acquired results.

\section{Basic Preliminaries}

The fractional calculus, with respect to another function, is discussed in this section. For more information, please see [1-4]. Let $J=[a, b] \subset \mathbb{R}$. Denote by $\mathcal{C}:=C(J, \mathbb{R})$ the Banach space of continuous real valued functions $\omega: J \rightarrow \mathbb{R}$ with the norm:

$$
\|\omega\|_{\infty}=\sup \{|\omega(\varkappa)|, \varkappa \in J\} .
$$

Now, we define the weighted space $C_{1-\gamma, \vartheta}(J, \mathbb{R})$ of function $\omega$ on $J$ by:

$$
\mathbb{C}_{1-\gamma}^{\vartheta}(J)=C_{1-\gamma, \vartheta}(J, \mathbb{R})=\left\{\omega:(a, b] \rightarrow \mathbb{R} ; \quad[\vartheta(\varkappa)-\vartheta(a)]^{1-\gamma} \omega(\varkappa) \in \mathcal{C}, 0 \leq \gamma<1\right\} .
$$

Obviously, $\mathbb{C}_{1-\gamma}^{\vartheta}(J)$ is a Banach space with the following norm:

$$
\|\omega\|_{\mathbb{C}_{1-\gamma}^{\vartheta}}=\left\|[\vartheta(\varkappa)-\vartheta(a)]^{1-\gamma} \omega(\varkappa)\right\|_{\mathcal{C}}=\max _{\varkappa \in J}\left|[\vartheta(\varkappa)-\vartheta(a)]^{1-\gamma} \omega(\varkappa)\right| .
$$

Let $\vartheta, \omega \in \mathcal{C}^{n}$ such that $\vartheta$ is increasing and $\vartheta^{\prime}(\varkappa) \neq 0$, for all $\varkappa \in J$.

Definition 1 ([12]). The $\vartheta$-Hilfer FD of a function $\omega$ of order $\varrho \in(n-1, n]$ and type $\beta \in[0,1]$ is defined by

$$
\mathcal{D}_{a+}^{\varrho, \beta ; \vartheta} \omega(\varkappa)=\mathcal{I}_{a+}^{\beta(n-\varrho) ; \vartheta} D_{\vartheta}^{n} \mathcal{I}_{a+}^{(1-\beta)(n-\varrho) ; \vartheta} \omega(\varkappa),
$$

where $n=[\varrho]+1, n \in \mathbb{N}$ and $D_{\vartheta}^{n}=\left(\frac{1}{\vartheta^{\prime}(\varkappa)} \frac{d}{d \varkappa}\right)^{n}$. The relation (5) can be written as

$$
\mathcal{D}_{a+}^{\varrho, \beta ; \vartheta} \omega(\varkappa)=\mathcal{I}_{a+}^{\gamma-\varrho ; \vartheta R L} \mathcal{D}_{a+}^{\gamma ; \vartheta} \omega(\varkappa),
$$

with $\gamma=\varrho+\beta(n-\varrho), \mathcal{I}_{a+}^{\gamma-\varrho ; \vartheta}(\cdot) a n d^{R L} \mathcal{D}_{a+}^{\gamma ; \vartheta}(\cdot)$ are called the $\vartheta-R L$ fractional integral and derivative defined by (see [1])

$$
\begin{aligned}
\mathcal{I}_{a+}^{\theta ; \vartheta} \omega(\varkappa)= & \frac{1}{\Gamma(\theta)} \int_{a}^{\varkappa} \vartheta^{\prime}(s)(\vartheta(\varkappa)-\vartheta(s))^{\theta-1} \omega(s) d s, \text { and } \\
& R L \mathcal{D}_{a+}^{\theta ; \vartheta} \omega(\varkappa)=D_{\vartheta}^{n} \mathcal{I}_{a+}^{n-\theta ; \vartheta} \omega(\varkappa),
\end{aligned}
$$


respectively. Furtheremore, the $\vartheta$-Caputo FD is given by (see [11]):

$$
{ }^{C} \mathcal{D}_{a+}^{\theta ; \vartheta} \omega(\varkappa)=\mathcal{I}_{a+}^{n-\theta ; \vartheta} D_{\vartheta}^{n} \omega(\varkappa),
$$

where $\theta \in\{\gamma-\varrho, \gamma, \varrho\}$.

Remark 1. In the case $\vartheta(\varkappa)=\varkappa$, the relations (5) and (7)-(9) reduce to the classical fractional operators in $[1,4,25]$.

Lemma 1 ([1,12]). Let $\varrho>0$ and $\beta>0$, and $\omega \in \mathcal{C}^{n}$. Then:

$$
\mathcal{I}_{a+}^{\varrho ; \vartheta} \mathcal{I}_{a+}^{\beta ; \vartheta} \omega(\varkappa)=\mathcal{I}_{a+}^{\varrho+\beta ; \vartheta} \omega(\varkappa)
$$

and

$$
\mathcal{D}_{a+}^{Q, \beta ; \vartheta} \mathcal{I}_{a+}^{\rho ; \vartheta} \omega(\varkappa)=\omega(\varkappa) .
$$

Lemma 2 ([1,12]). Let $\varrho, \beta>0$ and $\delta>0$. Then:

1. $\mathcal{I}_{a+}^{\varrho ; \vartheta}(\vartheta(\varkappa)-\vartheta(a))^{\delta-1}=\frac{\Gamma(\delta)}{\Gamma(\varrho+\delta)}(\vartheta(\varkappa)-\vartheta(a))^{\varrho+\delta-1}$,

2. $\mathcal{D}_{a+}^{\varrho, \beta ; \vartheta}(\vartheta(\varkappa)-\vartheta(a))^{\delta-1}=\frac{\Gamma(\delta)}{\Gamma(\delta-\varrho)}(\vartheta(\varkappa)-\vartheta(a))^{\varrho-\delta-1}, \delta>\gamma=\varrho+\beta(n-\varrho)$,

3. $\mathcal{D}_{a+}^{Q, \beta ; \vartheta}(\vartheta(\varkappa)-\vartheta(a))^{\delta-1}=0, \delta=\gamma$.

Lemma 3 ([12]). Assume that $\varrho \in(n-1, n](n \in \mathbb{N}), \beta \in[0,1]$ with $\gamma=\varrho+\beta(n-\varrho)$ and $\omega \in \mathcal{C}^{n}$. Then:

$$
\left(\mathcal{I}_{a^{+}}^{\varrho ; \vartheta} \mathcal{D}_{a^{+}}^{\varrho, \beta ; \vartheta} \omega\right)(\varkappa)=\omega(\varkappa)-\sum_{k=1}^{n} \frac{(\vartheta(\varkappa)-\vartheta(a))^{\gamma-k}}{\Gamma(\gamma-k+1)} D_{\vartheta}^{n}\left(\mathcal{I}_{a^{+}}^{(1-\beta)(n-\varrho) ; \vartheta} \omega\right)(a) .
$$

Particularly, if $1<\varrho \leq 2,0 \leq \beta \leq 1$ with $\gamma=\varrho+\beta(2-\varrho)$ we have:

$$
\begin{aligned}
\left(\mathcal{I}_{a^{+}}^{\rho ; \vartheta} \mathcal{D}_{a^{+}}^{\varrho, \beta ; \vartheta} \omega\right)(\varkappa)= & \omega(\varkappa)-\frac{(\vartheta(\varkappa)-\vartheta(a))^{\gamma-1}}{\Gamma(\gamma)}\left(\mathcal{I}_{a^{+}}^{1-\gamma ; \vartheta} \omega\right)(a) \\
& -\frac{(\vartheta(\varkappa)-\vartheta(a))^{\gamma-2}}{\Gamma(\gamma-1)}\left(\mathcal{I}_{a^{+}}^{2-\gamma ; \vartheta} \omega\right)(a) .
\end{aligned}
$$

Lemma 4 ([1]). Let $n-1 \leq \varrho<n$ and $\omega \in \mathcal{C}$. Then:

$$
\left(\mathcal{I}_{a+}^{\varrho ; \vartheta} \omega\right)(a)=\lim _{\varkappa \rightarrow a+}\left(\mathcal{I}_{a+}^{\varrho ; \vartheta} \omega\right)(\varkappa)=0 .
$$

Dhage's hybrid fixed point result $[26,27]$ will serve as the basis for demonstrating the existence of the solution to the problem (4).

Theorem $\mathbf{1}$ ([26,27]). Let $\mathbb{X} \subset \mathbb{C}_{1-\gamma}^{\vartheta}(J)$ be a convex, bounded, and closed set; there are operators: $\mathcal{P}, \mathcal{S}: \mathbb{C}_{1-\gamma}^{\vartheta}(J) \rightarrow \mathbb{C}_{1-\gamma}^{\vartheta}(J)$ and $\mathcal{Q}: \mathbb{X} \rightarrow \mathbb{C}_{1-\gamma}^{\vartheta}(J)$, such that:

(s1) $\mathcal{P}$ and $\mathcal{S}$ are Lipschitzian with Lipschitz constants $L_{\mathcal{P}}$ and $L_{\mathcal{S}}$, respectively;

(s2) $\mathcal{Q}$ is continuous and compact;

(s3) $u=\mathcal{P} u \mathcal{Q} v+\mathcal{S} u, \forall v \in \mathbb{X} \Rightarrow u \in \mathbb{X}$

(s4) $L_{\mathcal{P}} M_{\mathcal{Q}}+L_{\mathcal{S}}<1$, where $M_{\mathcal{Q}}=\|\mathcal{Q}(\mathbb{X})\|=\sup \{\|\mathcal{Q} v\|: v \in \mathbb{X}\}$.

Then, the operator equation $v=\mathcal{P} v \mathcal{Q} v+\mathcal{S} v$ possesses a solution in $\mathbb{X}$.

\section{Main Result}

The next lemma transacts with a linear-type of the problem (4). 
Lemma 5. Assume that $\varrho \in(1,2], \beta \in[0,1], \gamma=\varrho+\beta(2-\varrho), h(\varkappa) \in \mathbb{C}_{1-\gamma}^{\vartheta}(J)$, and

$$
\Lambda=\left[\omega_{1} \omega_{4}-\omega_{2} \omega_{3}\right] \neq 0 .
$$

A function $u \in \mathbb{C}_{1-\gamma}^{\vartheta}(J)$ is a solution of the following problem:

$$
\left\{\begin{array}{l}
\mathcal{D}_{a^{+}}^{\varrho, \beta ; \vartheta}\left(\frac{u(\varkappa)-f(\varkappa)}{g(\varkappa)}\right)=h(\varkappa), \varrho \in(1,2), \quad \varkappa \in J:=[a, b], \\
a_{1} \mathcal{I}_{a^{+}}^{1-\gamma ; \vartheta}\left(\frac{u(a)-f(a)}{g(a)}\right)+b_{1}\left(\frac{u(b)-f(b)}{g(b)}\right)=c_{1}, \\
a_{2} \mathcal{D}_{a^{+}}^{\sigma, \beta ; \vartheta}\left(\frac{u(\eta)-f(\eta)}{g(\eta)}\right)+b_{2} \mathcal{D}_{a^{+}}^{\sigma, \beta ; \vartheta}\left(\frac{u(b)-f(b)}{g(b)}\right)=c_{2}
\end{array}\right.
$$

if and only if:

$$
\begin{aligned}
u(\varkappa)= & g(\varkappa)\left[\mathcal{I}_{a^{+}}^{\varrho ; \vartheta} h(\varkappa)+\mu(\varkappa) b_{1} \mathcal{I}_{a^{+}}^{\varrho ; \vartheta} h(b)\right. \\
& -v(\varkappa)\left(a_{2} \mathcal{I}_{a^{+}}^{\varrho-\sigma ; \vartheta} h(\eta)+b_{2} \mathcal{I}_{a^{+}}^{Q-\sigma ; \vartheta} h(b)\right) \\
+ & \left.\Delta_{1}[\vartheta(\varkappa)-\vartheta(a)]^{\gamma-1}+\Delta_{2}[\vartheta(\varkappa)-\vartheta(a)]^{\gamma-2}\right]+f(\varkappa),
\end{aligned}
$$

where:

$$
\begin{aligned}
& \mu(\varkappa)=\frac{[\vartheta(\varkappa)-\vartheta(a)]^{\gamma-1}}{\Lambda \Gamma(\gamma)} \omega_{3}-\frac{[\vartheta(\varkappa)-\vartheta(a)]^{\gamma-2}}{\Lambda \Gamma(\gamma-1)} \omega_{4}, \\
& v(\varkappa)=\frac{[\vartheta(\varkappa)-\vartheta(a)]^{\gamma-1}}{\Lambda \Gamma(\gamma)} \omega_{1}-\frac{[\vartheta(\varkappa)-\vartheta(a)]^{\gamma-2}}{\Lambda \Gamma(\gamma-1)} \omega_{2},
\end{aligned}
$$

and

$$
\begin{aligned}
& \Delta_{1}=\frac{c_{1} \omega_{4}-c_{2} \omega_{2}}{\Lambda \Gamma(\gamma)} \\
& \Delta_{2}=\frac{c_{2} \omega_{1}-c_{1} \omega_{3}}{\Lambda \Gamma(\gamma-1)}
\end{aligned}
$$

along with

$$
\begin{gathered}
\omega_{1}=\left(\frac{a_{1}+b_{1}[\vartheta(\varkappa)-\vartheta(a)]^{\gamma-1}}{\Gamma(\gamma)}\right), \\
\omega_{2}=\left(\frac{b_{1}[\vartheta(\varkappa)-\vartheta(a)]^{\gamma-2}}{\Gamma(\gamma-1)}\right), \\
\omega_{3}=\left(\frac{a_{2}[\vartheta(\eta)-\vartheta(a)]^{\gamma-\sigma-1}+b_{2}[\vartheta(b)-\vartheta(a)]^{\gamma-\sigma-1}}{\Gamma(\gamma-\sigma)}\right), \\
\omega_{4}=\left(\frac{a_{2}[\vartheta(\eta)-\vartheta(a)]^{\gamma-\sigma-2}+b_{2}[\vartheta(b)-\vartheta(a)]^{\gamma-\sigma-2}}{\Gamma(\gamma-\sigma-1)}\right) .
\end{gathered}
$$

Proof. Let $u$ be a solution of the first equation of the problem (11). Applying the operator $\mathcal{I}_{a^{+}}^{Q ; \vartheta}$, by Lemma 3 and setting $\mathcal{I}_{a^{+}}^{2-\gamma ; \vartheta}\left(\frac{u(a)-f(a)}{g(a)}\right)=k_{1}, \mathcal{I}_{a^{+}}^{1-\gamma ; \vartheta}\left(\frac{u(a)-f(a)}{g(a)}\right)=k_{2}$, we have:

$$
\left(\frac{u(\varkappa)-f(\varkappa)}{g(\varkappa)}\right)=\frac{k_{2}}{\Gamma(\gamma)}[\vartheta(\varkappa)-\vartheta(a)]^{\gamma-1}+\frac{k_{1}}{\Gamma(\gamma-1)}[\vartheta(\varkappa)-\vartheta(a)]^{\gamma-2}+\mathcal{I}_{a^{+}}^{\varrho ; \vartheta} h(\varkappa) .
$$

Applying the boundary conditions in Equation (21), and after collecting the similar terms in one part, we have the following equations:

$$
c_{1}-b_{1} \mathcal{I}_{a^{+}}^{\varrho ; \vartheta} h(b)=k_{2}\left(\frac{a_{1}+b_{1}[\vartheta(b)-\vartheta(a)]^{\gamma-1}}{\Gamma(\gamma)}\right)+k_{1}\left(\frac{b_{1}[\vartheta(b)-\vartheta(a)]^{\gamma-2}}{\Gamma(\gamma-1)}\right),
$$

and 


$$
\begin{aligned}
c_{2}-a_{2} \mathcal{I}_{a^{+}}^{Q-\sigma ; \vartheta} h(\eta)-b_{2} \mathcal{I}_{a^{+}}^{Q-\sigma ; \vartheta} h(b) & =k_{2}\left(\frac{a_{2}[\vartheta(\eta)-\vartheta(a)]^{\gamma-\sigma-1}+b_{2}[\vartheta(b)-\vartheta(a)]^{\gamma-\sigma-1}}{\Gamma(\gamma-\sigma)}\right) \\
& +k_{1}\left(\frac{a_{2}[\vartheta(\eta)-\vartheta(a)]^{\gamma-\sigma-2}+b_{2}[\vartheta(b)-\vartheta(a)]^{\gamma-\sigma-2}}{\Gamma(\gamma-\sigma-1)}\right) .
\end{aligned}
$$

Rewriting Equations (22) and (23), by using Equations (17)-(20), we obtain:

$$
c_{1}-b_{1} \mathcal{I}_{a^{+}}^{Q ; \vartheta} h(b)=k_{2} \omega_{1}+k_{1} \omega_{2}
$$

and

$$
c_{2}-a_{2} \mathcal{I}_{a^{+}}^{Q-\sigma ; \vartheta} h(\eta)-b_{2} \mathcal{I}_{a^{+}}^{Q-\sigma ; \vartheta} h(b)=k_{2} \omega_{3}+k_{1} \omega_{4} .
$$

which, on solving, yields:

$$
k_{1}=\frac{1}{\Lambda}\left\{c_{2} \omega_{1}-c_{1} \omega_{3}+b_{1} \omega_{3} \mathcal{I}_{a^{+}}^{Q ; \vartheta} h(b)-a_{2} \omega_{1} \mathcal{I}_{a^{+}}^{\varrho-\sigma ; \vartheta} h(\eta)-b_{2} \omega_{1} \mathcal{I}_{a^{+}}^{\varrho-\sigma ; \vartheta} h(b)\right\},
$$

and

$$
k_{2}=\frac{1}{\Lambda}\left\{c_{1} \omega_{4}-c_{2} \omega_{2}-b_{1} \omega_{4} \mathcal{I}_{a^{+}}^{\rho ; \vartheta} h(b)+a_{2} \omega_{2} \mathcal{I}_{a^{+}}^{\varrho-\sigma ; \vartheta} h(\eta)-b_{2} \omega_{2} \mathcal{I}_{a^{+}}^{\varrho-\sigma ; \vartheta} h(b)\right\} .
$$

Substituting the value of $k_{1}$ and $k_{2}$ in Equation (32), we get the Equation (12). Conversly, assume that $u$ is a solution of Equation (12). Then, we get

$$
\begin{aligned}
\frac{u(\varkappa)-f(\varkappa)}{g(\varkappa)}= & {\left[\mathcal{I}_{a^{+}}^{\varrho ; \vartheta} h(\varkappa)+\mu(\varkappa) b_{1} \mathcal{I}_{a^{+}}^{\varrho ; \vartheta} h(b)\right.} \\
& -v(\varkappa)\left(a_{2} \mathcal{I}_{a^{+}}^{\varrho-\sigma ; \vartheta} h(\eta)+b_{2} \mathcal{I}_{a^{+}}^{\varrho-\sigma ; \vartheta} h(b)\right) \\
+ & \left.\Delta_{1}[\vartheta(\varkappa)-\vartheta(a)]^{\gamma-1}+\Delta_{2}[\vartheta(\varkappa)-\vartheta(a)]^{\gamma-2}\right] .
\end{aligned}
$$

Taking the operator $\mathcal{D}_{a^{+}}^{Q, \beta ; \vartheta}$ on both sides of above equation, with the help of Definition 1 and Lemmas 1-3, we get:

$$
\mathcal{D}_{a^{+}}^{Q, \beta ; \vartheta}\left(\frac{u(\varkappa)-f(\varkappa)}{g(\varkappa)}\right)=h(\varkappa) .
$$

On the other hand, applying the operator $\mathcal{I}_{a^{+}}^{1-\gamma ; \vartheta}$ on both sides of Equation (12), with replace $\varkappa$ by $a$, we get:

$$
\begin{aligned}
\mathcal{I}_{a^{+}}^{1-\gamma ; \vartheta}\left(\frac{u(a)-f(a)}{g(a)}\right)= & \mathcal{I}_{a^{+}}^{1-\gamma ; \vartheta}\left[\mathcal{I}_{a^{+}}^{\varrho ; \vartheta} h(a)+\mu(a) b_{1} \mathcal{I}_{a^{+}}^{Q ; \vartheta} h(b)\right. \\
& \left.-v(a)\left(a_{2} \mathcal{I}_{a^{+}}^{Q-\sigma ; \vartheta} h(\eta)+b_{2} \mathcal{I}_{a^{+}}^{Q-\sigma ; \vartheta} h(b)\right)\right] .
\end{aligned}
$$

Next, replace $\varkappa$ by $a$ in Equation (12), we get

$$
\begin{aligned}
\left(\frac{u(b)-f(b)}{g(b)}\right)= & {\left[\mathcal{I}_{a^{+}}^{\varrho ; \vartheta} h(b)+\mu(b) b_{1} \mathcal{I}_{a^{+}}^{Q ; \vartheta} h(b)\right.} \\
& -v(b)\left(a_{2} \mathcal{I}_{a^{+}}^{Q-\sigma ; \vartheta} h(\eta)+b_{2} \mathcal{I}_{a^{+}}^{Q-\sigma ; \vartheta} h(b)\right) \\
+ & \left.\Delta_{1}[\vartheta(b)-\vartheta(a)]^{\gamma-1}+\Delta_{2}[\vartheta(b)-\vartheta(a)]^{\gamma-2}\right] .
\end{aligned}
$$


Multiply Equation (27) by $a_{1}$ and Equation (28) by $b_{1}$ and we get:

$$
\begin{aligned}
& a_{1} \mathcal{I}_{a^{+}}^{1-\gamma ; \vartheta}\left(\frac{u(a)-f(a)}{g(a)}\right)+b_{1}\left(\frac{u(b)-f(b)}{g(b)}\right) \\
= & a_{1} \mathcal{I}_{a^{+}}^{1-\gamma ; \vartheta}\left[\mathcal{I}_{a^{+}}^{Q ; \vartheta} h(a)+\mu(a) b_{1} \mathcal{I}_{a^{+}}^{Q ; \vartheta} h(b)\right. \\
& \left.-v(a)\left(a_{2} \mathcal{I}_{a^{+}}^{Q-\sigma ; \vartheta} h(\eta)+b_{2} \mathcal{I}_{a^{+}}^{Q-\sigma ; \vartheta} h(b)\right)\right] \\
& +b_{1}\left[\mathcal{I}_{a^{+}}^{\varrho ; \vartheta} h(b)+\mu(b) b_{1} \mathcal{I}_{a^{+}}^{Q ; \vartheta} h(b)\right. \\
& -v(b)\left(a_{2} \mathcal{I}_{a^{+}}^{Q-\sigma ; \vartheta} h(\eta)+b_{2} \mathcal{I}_{a^{+}}^{Q-\sigma ; \vartheta} h(b)\right) \\
& \left.+\Delta_{1}[\vartheta(b)-\vartheta(a)]^{\gamma-1}+\Delta_{2}[\vartheta(b)-\vartheta(a)]^{\gamma-2}\right] .
\end{aligned}
$$

By the relations (13)-(20), one can get the first condition in the problem (11). On the other hand, applying the operator $\mathcal{D}_{a^{+}}^{\sigma, \beta ; \vartheta}$ on both sides of Equation (12), by replacing $\varkappa$ by $\eta$, we get:

$$
\begin{aligned}
\mathcal{D}_{a^{+}}^{\sigma, \beta ; \vartheta}\left(\frac{u(\eta)-f(\eta)}{g(\eta)}\right)= & \mathcal{D}_{a^{+}}^{\sigma, \beta ; \vartheta}\left[\mathcal{I}_{a^{+}}^{\rho ; \vartheta} h(\eta)+\mu(\eta) b_{1} \mathcal{I}_{a^{+}}^{\rho ; \vartheta} h(b)\right. \\
& \left.-v(\eta)\left(a_{2} \mathcal{I}_{a^{+}}^{\rho-\sigma ; \vartheta} h(\eta)+b_{2} \mathcal{I}_{a^{+}}^{\varrho-\sigma ; \vartheta} h(b)\right)\right] .
\end{aligned}
$$

Again, applying the operator $\mathcal{D}_{a^{+}}^{\sigma, \beta ; \vartheta}$ on both sides of Equation (12), by replacing $\varkappa$ by $b$, we get:

$$
\begin{aligned}
\mathcal{D}_{a^{+}}^{\sigma, \beta ; \vartheta}\left(\frac{u(b)-f(b)}{g(b)}\right)= & \mathcal{D}_{a^{+}}^{\sigma, \beta ; \vartheta}\left[\mathcal{I}_{a^{+}}^{\varrho ; \vartheta} h(b)+\mu(b) b_{1} \mathcal{I}_{a^{+}}^{\rho ; \vartheta} h(b)\right. \\
& -v(b)\left(a_{2} \mathcal{I}_{a^{+}}^{\varrho-\sigma ; \vartheta} h(\eta)+b_{2} \mathcal{I}_{a^{+}}^{\varrho-\sigma ; \vartheta} h(b)\right) \\
+ & \left.\Delta_{1}[\vartheta(b)-\vartheta(a)]^{\gamma-1}+\Delta_{2}[\vartheta(b)-\vartheta(a)]^{\gamma-2}\right] .
\end{aligned}
$$

Multiply Equation (29) by $a_{1}$ and Equation (30) by $b_{1}$, we get

$$
\begin{aligned}
& a_{1} \mathcal{D}_{a^{+}}^{\sigma, \beta ; \vartheta}\left(\frac{u(\eta)-f(\eta)}{g(\eta)}\right)+b_{1} \mathcal{D}_{a^{+}}^{\sigma, \beta ; \vartheta}\left(\frac{u(b)-f(b)}{g(b)}\right) \\
= & a_{1} \mathcal{D}_{a^{+}}^{\sigma, \beta ; \vartheta}\left[\mathcal{I}_{a^{+}}^{Q ; \vartheta} h(\eta)+\mu(\eta) b_{1} \mathcal{I}_{a^{+}}^{\varrho ; \vartheta} h(b)\right. \\
& \left.-v(\eta)\left(a_{2} \mathcal{I}_{a^{+}}^{Q-\sigma ; \vartheta} h(\eta)+b_{2} \mathcal{I}_{a^{+}}^{Q-\sigma ; \vartheta} h(b)\right)\right] \\
& +b_{1} \mathcal{D}_{a^{+}}^{\sigma, \beta ; \vartheta}\left[\mathcal{I}_{a^{+}}^{\varrho ; \vartheta} h(b)+\mu(b) b_{1} \mathcal{I}_{a^{+}}^{\rho ; \vartheta} h(b)\right. \\
& -v(b)\left(a_{2} \mathcal{I}_{a^{+}}^{Q-\sigma ; \vartheta} h(\eta)+b_{2} \mathcal{I}_{a^{+}}^{Q-\sigma ; \vartheta} h(b)\right) \\
& \left.+\Delta_{1}[\vartheta(b)-\vartheta(a)]^{\gamma-1}+\Delta_{2}[\vartheta(b)-\vartheta(a)]^{\gamma-2}\right] .
\end{aligned}
$$

By simple calculations, with the help of Equations (13)-(20), one can get the second condition in the Equation (11).

As a starting point, we must make the following assumptions, in order to verify the main results in the sequel:

$(\mathbb{H} Y \mathbb{P} 1)$ there exists $L_{\mathcal{G}}>0$ and $L_{\mathcal{S}}>0$, such that:

$$
|\mathcal{F}(\varkappa, u)-\mathcal{F}(\varkappa, v)| \leq L_{\mathcal{S}}|u-v|,
$$


and

$$
|\mathcal{G}(\varkappa, u)-\mathcal{G}(\varkappa, v)| \leq[\vartheta(\varkappa)-\vartheta(a)]^{1-\gamma} L_{\mathcal{G}}|u-v|,
$$

for all $\varkappa \in J$ and $u, v \in \mathbb{R}$.

$(\mathbb{H} Y \mathbb{P} 2)$ There exists bounded functions $p, q, r: J \rightarrow \mathbb{R}_{+}$, such that:

$$
|\mathcal{H}(\varkappa, u, v)| \leq p(\varkappa)+q(\varkappa)|u|+r(\varkappa)|v|,
$$

for $\varkappa \in J$ and $u, v \in \mathbb{R}$.

$(\mathbb{H} \mathbb{Y} \mathbb{P} 3)$ There exist $\mathcal{F}^{*}>0$ and $\mathcal{G}^{*}>0$ such that

$$
\mathcal{F}^{*}=\sup _{\varkappa \in J}|\mathcal{F}(\varkappa, 0)|, \quad \mathcal{G}^{*}=\sup _{\varkappa \in J}|\mathcal{G}(\varkappa, 0)|,
$$

along with

$$
p^{*}=\sup _{\varkappa \in J} p(\varkappa), q^{*}=\sup _{\varkappa \in J} q(\varkappa), r^{*}=\sup _{\varkappa \in J} r(\varkappa)<1 .
$$

$(\mathbb{H} Y \mathbb{P} 4)$ The following inequality holds:

$$
\left[M_{\mathcal{Q}} L_{\mathcal{P}}+L_{\mathcal{S}}\right]<1,
$$

where:

$$
\begin{aligned}
M_{\mathcal{Q}} & =\mathcal{A} \Phi+\Delta, \\
\mathcal{A} & =\frac{[\vartheta(b)-\vartheta(a)]^{1-\gamma+\varrho}}{\Gamma(\varrho+1)}+\frac{[\vartheta(b)-\vartheta(a)]^{\varrho}}{\Gamma(\varrho+1)} \bar{\mu}(b) b_{1} \\
& -\bar{v}(b)\left(\frac{[\vartheta(\eta)-\vartheta(a)]^{Q-\sigma}}{\Gamma(\varrho-\sigma+1)} a_{2}+\frac{[\vartheta(b)-\vartheta(a)]^{Q-\sigma}}{\Gamma(\varrho-\sigma+1)} b_{2}\right), \\
\bar{\mu}(\varkappa) & =[\vartheta(\varkappa)-\vartheta(a)]^{1-\gamma} \mu(\varkappa), \quad \bar{v}(\varkappa)=[\vartheta(\varkappa)-\vartheta(a)]^{1-\gamma} v(\varkappa) \\
\Phi & =\frac{[\vartheta(b)-\vartheta(a)]^{1-\gamma} p^{*}+q^{*} \rho}{1-r^{*}} \\
\Delta & =\Delta_{1}+\Delta_{2}[\vartheta(b)-\vartheta(a)]^{-1}, \\
L_{\mathcal{P}} & =[\vartheta(b)-\vartheta(a)]^{1-\gamma} L_{\mathcal{G}}, \\
M_{\mathcal{Q}} & =\mathcal{A} \Phi+\Delta .
\end{aligned}
$$

To show the existence of solutions of the proposed system (4), we will need to prove the theorem below.

Theorem 2. Suppose that the hypotheses ( $\mathbb{H} Y \mathbb{P} 1)$-( $(\mathbb{H} Y \mathbb{P} 4)$ are satisfied. If

$$
L_{\mathcal{P}} M_{\mathcal{Q}}+L_{\mathcal{S}}<1
$$

then the problem (4) has a solution on J.

Proof. In view of Lemma 5, the solution of the problem (4) is given by:

$$
\begin{aligned}
u(\varkappa)= & \mathcal{G}(\varkappa, u(\varkappa))\left[\mathcal{I}_{a^{+}}^{\varrho ; \vartheta} \mathcal{H}_{u}(\varkappa)+\mu(\varkappa) b_{1} \mathcal{I}_{a^{+}}^{\varrho ; \vartheta} \mathcal{H}_{u}(b)\right. \\
& -v(\varkappa)\left(a_{2} \mathcal{I}_{a^{+}}^{Q-\sigma ; \vartheta} \mathcal{H}_{u}(\eta)+b_{2} \mathcal{I}_{a^{+}}^{Q-\sigma ; \vartheta} \mathcal{H}_{u}(b)\right) \\
+ & \left.\Delta_{1}[\vartheta(\varkappa)-\vartheta(a)]^{\gamma-1}+\Delta_{2}[\vartheta(\varkappa)-\vartheta(a)]^{\gamma-2}\right]+\mathcal{F}(\varkappa, u(\varkappa)),
\end{aligned}
$$

where: $\mathcal{H}_{u}(\varkappa):=\mathcal{H}\left(\varkappa, u(\varkappa), \mathcal{D}_{a^{+}}^{\varrho, \beta ; \vartheta}\left(\frac{u(\varkappa)-\mathcal{F}(\varkappa, u(\varkappa))}{\mathcal{G}(\varkappa, u(\varkappa))}\right)\right)$. 
Choose $\rho>0$, so that:

$$
\rho \geq \frac{(\mathcal{A} \Phi+\Delta) \mathcal{G}^{*}+\mathcal{F}^{*}}{1-\left[(\mathcal{A} \Phi+\Delta) L_{\mathcal{P}}+L_{\mathcal{S}}\right]} .
$$

Define a closed ball, $\mathbb{X} \subset \mathbb{C}_{1-\gamma}^{\vartheta}(J)$, by:

$$
\mathbb{X}=\left\{u \in \mathbb{C}_{1-\gamma}^{\vartheta}(J):\|u\|_{\mathbb{C}_{1-\gamma}^{\vartheta}} \leq \rho\right\}
$$

and let the operators $\mathcal{P}: \mathbb{C}_{1-\gamma}^{\vartheta}(J) \rightarrow \mathbb{C}_{1-\gamma}^{\vartheta}(J), \mathcal{S}: \mathbb{C}_{1-\gamma}^{\vartheta}(J) \rightarrow \mathbb{C}_{1-\gamma}^{\vartheta}(J)$ and $\mathcal{Q}: \mathbb{X} \rightarrow$ $\mathbb{C}_{1-\gamma}^{\vartheta}(J)$ be defined by:

$$
\mathcal{P} u=\mathcal{G}(\varkappa, u(\varkappa)), \quad \varkappa \in J,
$$

$$
\begin{aligned}
\mathcal{Q} u & =\mathcal{I}_{a^{+}}^{\varrho ; \vartheta} \mathcal{H}_{u}(\varkappa)+\mu(\varkappa) b_{1} \mathcal{I}_{a^{+}}^{\varrho ; \vartheta} \mathcal{H}_{u}(b)-v(\varkappa)\left(a_{2} \mathcal{I}_{a^{+}}^{\varrho-\sigma ; \vartheta} \mathcal{H}_{u}(\eta)\right. \\
& \left.+b_{2} \mathcal{I}_{a^{+}}^{\varrho-\sigma ; \vartheta} \mathcal{H}_{u}(b)\right)+\Delta_{1}[\vartheta(\varkappa)-\vartheta(a)]^{\gamma-1}+\Delta_{2}[\vartheta(\varkappa)-\vartheta(a)]^{\gamma-2}, \quad \varkappa \in J,
\end{aligned}
$$

and

$$
\mathcal{S} u=\mathcal{F}(\varkappa, u(\varkappa)), \quad \varkappa \in J .
$$

Then, the Equation (32) can be written as:

$$
\mathcal{P} u(\varkappa) \mathcal{Q} u(\varkappa)+\mathcal{S} u(\varkappa)=u(\varkappa), \quad \varkappa \in J .
$$

Now, we need to show that the operators $\mathcal{P}, \mathcal{Q}$, and $\mathcal{S}$ agree with the assumptions of Dhage's Theorem 1.

Step-I: We first show that $\mathcal{P}$ and $\mathcal{S}$ are Lipschitzian on $\mathbb{C}_{1-\gamma}^{\vartheta}(J)$, with Lipschitz constants $L_{\mathcal{P}}$ and $L_{\mathcal{S}}$, respectively. Let $u, \bar{u} \in \mathbb{C}_{1-\gamma}^{\vartheta}(J)$. Then, using $(\mathbb{H} \mathbb{Y} \mathbb{P} 2)$, we have:

$$
\begin{aligned}
\left|(\mathcal{P} u(\varkappa)-\mathcal{P} \bar{u}(\varkappa))[\vartheta(\varkappa)-\vartheta(a)]^{1-\gamma}\right| & =[\vartheta(\varkappa)-\vartheta(a)]^{1-\gamma}|\mathcal{G}(\varkappa, u(\varkappa))-\mathcal{G}(\varkappa, \bar{u}(\varkappa))| \\
& \leq[\vartheta(\varkappa)-\vartheta(a)]^{1-\gamma} L_{\mathcal{G}}[\vartheta(\varkappa)-\vartheta(a)]^{1-\gamma}|u(\varkappa)-\bar{u}(\varkappa)| \\
& \leq[\vartheta(b)-\vartheta(a)]^{1-\gamma} L_{\mathcal{G}}\|u-\bar{u}\|_{\mathbb{C}_{1-\gamma}^{\vartheta}},
\end{aligned}
$$

for all $\varkappa \in J$. Operating the supremum norm over $\varkappa$, we get that:

$$
\|\mathcal{P} u-\mathcal{P}(\bar{u})\|_{\mathbb{C}_{1-\gamma}^{\vartheta}} \leq L_{\mathcal{P}}\|u-\bar{u}\|_{\mathbb{C}_{1-\gamma}^{\theta}}
$$

for all $u, \bar{u} \in \mathbb{C}_{1-\gamma}^{\vartheta}(J)$. In consequence, $\mathcal{P}$ is a Lipschitz map subject to constant:

$$
L_{\mathcal{P}}=[\vartheta(b)-\vartheta(a)]^{1-\gamma} L_{\mathcal{G}}
$$

By the same way and using $(\mathbb{H} \mathbb{Y} \mathbb{P} 3)$, we get that:

$$
\begin{aligned}
\left|(\mathcal{S} u(\varkappa)-\mathcal{S} \bar{u}(\varkappa))[\vartheta(\varkappa)-\vartheta(a)]^{1-\gamma}\right| & =[\vartheta(\varkappa)-\vartheta(a)]^{1-\gamma}|\mathcal{F}(\varkappa, u(\varkappa))-\mathcal{F}(\varkappa, \bar{u}(\varkappa))| \\
& \leq[\vartheta(\varkappa)-\vartheta(a)]^{1-\gamma} L_{\mathcal{S}}|u(\varkappa)-\bar{u}(\varkappa)| \\
& \leq L_{\mathcal{S}}\|u-\bar{u}\|_{\mathbb{C}_{1-\gamma}^{\vartheta}}
\end{aligned}
$$

for all $\varkappa \in J$. Operating the supremum norm over $\varkappa$, we get that:

$$
\|\mathcal{S} u-\mathcal{S}(\bar{u})\|_{\mathbb{C}_{1-\gamma}^{\vartheta}} \leq L_{\mathcal{S}}\|u-\bar{u}\|_{\mathbb{C}_{1-\gamma}^{\vartheta}}
$$


for all $u, \bar{u} \in \mathbb{C}_{1-\gamma}^{\vartheta}(J)$. In consequence, $\mathcal{S}$ is a Lipschitz map subject to constant $L_{\mathcal{S}}$.

Step-II: We show that $\mathcal{Q}$ is completely continuous operator from $\mathbb{X}$ into $\mathbb{C}_{1-\gamma}^{\vartheta}(J)$. To achieve the continuity of $\mathcal{Q}$, we need to prove that the sequence $\left\{u_{n}\right\}_{n \in \mathbb{N}} \rightarrow u \in \mathbb{X}$. Then, Lebesgue's dominated convergence result yields:

$$
\begin{aligned}
& \lim _{n \rightarrow \infty} \mathcal{Q} u_{n}(\varkappa)=\lim _{n \rightarrow \infty} \frac{1}{\Gamma(\varrho)} \int_{a}^{\varkappa} \vartheta^{\prime}(s)(\vartheta(\varkappa)-\vartheta(s))^{\varrho-1} \mathcal{H}_{u n}(s) d s \\
& -\lim _{n \rightarrow \infty} \mu(\varkappa) b_{1} \frac{1}{\Gamma(\varrho)} \int_{a}^{b} \vartheta^{\prime}(s)(\vartheta(b)-\vartheta(s))^{\varrho-1} \mathcal{H}_{u_{n}}(s) d s \\
& -\lim _{n \rightarrow \infty} v(\varkappa)\left(a_{2} \frac{1}{\Gamma(\varrho-\sigma)} \int_{a}^{\eta} \vartheta^{\prime}(s)(\vartheta(\eta)-\vartheta(s))^{\varrho-\sigma-1} \mathcal{H}_{u_{n}}(s) d s\right. \\
& \left.+a_{2} \frac{1}{\Gamma(\varrho-\sigma)} \int_{a}^{b} \vartheta^{\prime}(s)(\vartheta(b)-\vartheta(s))^{\varrho-\sigma-1} \mathcal{H}_{u_{n}}(s) d s\right) \\
& +\Delta_{1}[\vartheta(b)-\vartheta(a)]^{\gamma-1}+\Delta_{2}[\vartheta(\varkappa)-\vartheta(a)]^{\gamma-2} \\
& =\frac{1}{\Gamma(\varrho)} \int_{a}^{\varkappa} \vartheta^{\prime}(s)(\vartheta(\varkappa)-\vartheta(s))^{\varrho-1} \lim _{n \rightarrow \infty} \mathcal{H}_{u n}(s) d s \\
& -\mu(\varkappa) b_{1} \frac{1}{\Gamma(\varrho)} \int_{a}^{b} \vartheta^{\prime}(s)(\vartheta(b)-\vartheta(s))^{\varrho-1} \lim _{n \rightarrow \infty} \mathcal{H}_{u_{n}}(s) d s \\
& -v(\varkappa)\left(a_{2} \frac{1}{\Gamma(\varrho-\sigma)} \int_{a}^{\eta} \vartheta^{\prime}(s)(\vartheta(\eta)-\vartheta(s))^{\varrho-\sigma-1} \lim _{n \rightarrow \infty} \mathcal{H}_{u_{n}}(s) d s\right. \\
& \left.+a_{2} \frac{1}{\Gamma(\varrho-\sigma)} \int_{a}^{b} \vartheta^{\prime}(s)(\vartheta(b)-\vartheta(s))^{\varrho-\sigma-1} \lim _{n \rightarrow \infty} \mathcal{H}_{u_{n}}(s) d s\right) \\
& +\Delta_{1}[\vartheta(b)-\vartheta(a)]^{\gamma-1}+\Delta_{2}[\vartheta(\varkappa)-\vartheta(a)]^{\gamma-2} \\
& =\frac{1}{\Gamma(\varrho)} \int_{a}^{\varkappa} \vartheta^{\prime}(s)(\vartheta(\varkappa)-\vartheta(s))^{\varrho-1} \mathcal{H}_{u}(s) d s \\
& -\mu(\varkappa) b_{1} \frac{1}{\Gamma(\varrho)} \int_{a}^{b} \vartheta^{\prime}(s)(\vartheta(b)-\vartheta(s))^{\varrho-1} \mathcal{H}_{u}(s) d s \\
& -v(\varkappa)\left(a_{2} \frac{1}{\Gamma(\varrho-\sigma)} \int_{a}^{\eta} \vartheta^{\prime}(s)(\vartheta(\eta)-\vartheta(s))^{\varrho-\sigma-1} \mathcal{H}_{u}(s) d s\right. \\
& \left.+a_{2} \frac{1}{\Gamma(\varrho-\sigma)} \int_{a}^{b} \vartheta^{\prime}(s)(\vartheta(b)-\vartheta(s))^{\varrho-\sigma-1} \mathcal{H}_{u}(s) d s\right) \\
& +\Delta_{1}[\vartheta(\varkappa)-\vartheta(a)]^{\gamma-1}+\Delta_{2}[\vartheta(\varkappa)-\vartheta(a)]^{\gamma-2} \\
& =\mathcal{Q} u(\varkappa) \text {, }
\end{aligned}
$$

where: $\mathcal{H}_{u_{n}}, \mathcal{H}_{u} \in \mathbb{C}_{1-\gamma}^{\vartheta}(J)$, such that

$$
\mathcal{H}_{u_{n}}(\varkappa)=\mathcal{H}\left(\varkappa, u(\varkappa), \mathcal{H}_{u_{n}}(\varkappa)\right) \text { and } \mathcal{H}_{u}(\varkappa)=\mathcal{H}\left(\varkappa, u(\varkappa), \mathcal{H}_{u}(\varkappa)\right) \text {, }
$$

for all $\varkappa \in J$. Hence, $\mathcal{Q} u_{n}$ converges to $\mathcal{Q} u$ pointwise on $J$.

Next, we will show that the operator $\mathcal{Q}$ is compact on $\mathbb{X}$. Firstly, to ensure the uniform boundedness, let $u \in \mathbb{X}$ (and applying $(\mathbb{H} \mathbb{Y} \mathbb{P} 3)$ ), we get:

$$
\begin{aligned}
\left|[\vartheta(\varkappa)-\vartheta(a)]^{1-\gamma} \mathcal{H}_{u}(\varkappa)\right| & =\left|[\vartheta(\varkappa)-\vartheta(a)]^{1-\gamma} \mathcal{H}\left(\varkappa, u(\varkappa), \mathcal{H}_{u}(\varkappa)\right)\right| \\
& \leq[\vartheta(\varkappa)-\vartheta(a)]^{1-\gamma}\left(p(\varkappa)+q(\varkappa)|u(\varkappa)|+r(\varkappa)\left|\mathcal{G}_{u}(\varkappa)\right|\right) \\
& \leq[\vartheta(b)-\vartheta(a)]^{1-\gamma} p^{*}+q^{*} \rho+\lambda_{3}^{*}\left|[\vartheta(\varkappa)-\vartheta(a)]^{1-\gamma} \mathcal{G}_{u}(\varkappa)\right| .
\end{aligned}
$$


Hence:

$$
\left|[\vartheta(\varkappa)-\vartheta(a)]^{1-\gamma} \mathcal{H}_{u}(\varkappa)\right| \leq \frac{[\vartheta(b)-\vartheta(a)]^{1-\gamma} p^{*}+q^{*} \rho}{1-r^{*}} .
$$

Then, we have:

$$
\sup _{\varkappa \in(a, b]}\left|[\vartheta(\varkappa)-\vartheta(a)]^{1-\gamma} \mathcal{H}_{u}(\varkappa)\right| \leq \frac{[\vartheta(b)-\vartheta(a)]^{1-\gamma} p^{*}+q^{*} \rho}{1-r^{*}}=\Phi .
$$

For $\varkappa \in(a, b]$, we have:

$$
\begin{aligned}
& \left|(\mathcal{Q} u(\varkappa))[\vartheta(\varkappa)-\vartheta(a)]^{1-\gamma}\right| \\
& \leq[\vartheta(\varkappa)-\vartheta(a)]^{1-\gamma} \frac{1}{\Gamma(\varrho)} \int_{a}^{\varkappa} \vartheta^{\prime}(s)(\vartheta(\varkappa)-\vartheta(s))^{\varrho-1}\left|\mathcal{H}_{u}(s)\right| d s \\
& +[\vartheta(\varkappa)-\vartheta(a)]^{1-\gamma}|\mu(\varkappa)| b_{1} \frac{1}{\Gamma(\varrho)} \int_{a}^{b} \vartheta^{\prime}(s)(\vartheta(b)-\vartheta(s))^{\varrho-1}\left|\mathcal{H}_{u}(s)\right| d s \\
& +[\vartheta(\varkappa)-\vartheta(a)]^{1-\gamma}|v(\varkappa)|\left(\frac{a_{2}}{\Gamma(\varrho-\sigma)} \int_{a}^{\eta} \vartheta^{\prime}(s)(\vartheta(\eta)-\vartheta(s))^{\varrho-\sigma-1}\left|\mathcal{H}_{u}(s)\right| d s\right. \\
& \left.+\frac{a_{2}}{\Gamma(\varrho-\sigma)} \int_{a}^{b} \vartheta^{\prime}(s)(\vartheta(b)-\vartheta(s))^{\varrho-\sigma-1}\left|\mathcal{H}_{u}(s)\right| d s\right) \\
& +[\vartheta(\varkappa)-\vartheta(a)]^{1-\gamma}\left|\Delta_{1}\right|[\vartheta(\varkappa)-\vartheta(a)]^{\gamma-1}+[\vartheta(\varkappa)-\vartheta(a)]^{1-\gamma}\left|\Delta_{2}\right|[\vartheta(\varkappa)-\vartheta(a)]^{\gamma-2} \\
& \leq \frac{[\vartheta(b)-\vartheta(a)]^{1-\gamma+\varrho}}{\Gamma(\varrho+1)} \Phi+\frac{[\vartheta(b)-\vartheta(a)]^{\varrho}}{\Gamma(\varrho+1)} \Phi \bar{\mu}(b) b_{1} \\
& -\Phi \bar{v}(b)\left(\frac{[\vartheta(\eta)-\vartheta(a)]^{\varrho-\sigma}}{\Gamma(\varrho-\sigma+1)} a_{2}+\frac{[\vartheta(b)-\vartheta(a)]^{\varrho-\sigma}}{\Gamma(\varrho-\sigma+1)} b_{2}\right) \\
& +\left|\Delta_{1}\right|+\left|\Delta_{2}\right| .
\end{aligned}
$$

Taking the supremum in terms of $\varkappa$ in above, we get:

$$
\begin{aligned}
\|\mathcal{Q} u(\varkappa)\|_{\mathbb{C}_{1-\gamma}^{\vartheta}} & \leq \frac{[\vartheta(b)-\vartheta(a)]^{1-\gamma+\varrho}}{\Gamma(\varrho+1)} \Phi+\frac{[\vartheta(b)-\vartheta(a)]^{\varrho}}{\Gamma(\varrho+1)} \Phi \bar{\mu}(b) b_{1} \\
& -\Phi \bar{v}(b)\left(\frac{[\vartheta(\eta)-\vartheta(a)]^{\varrho-\sigma}}{\Gamma(\varrho-\sigma+1)} a_{2}+\frac{[\vartheta(b)-\vartheta(a)]^{\varrho-\sigma}}{\Gamma(\varrho-\sigma+1)} b_{2}\right) \\
& +\left|\Delta_{1}\right|+\left|\Delta_{2}\right|[\vartheta(b)-\vartheta(a)]^{-1} \\
& =\mathcal{A} \Phi+\Delta<\infty,
\end{aligned}
$$

for all $u \in \mathbb{X}$. Thus $\mathcal{Q}$ is a uniformly bounded operator on $\mathbb{X}$.

Secondly, we shall show that $\mathcal{Q}$ is equicontinuous. Let $u \in \mathbb{X}$ and let $\varkappa_{1}, \varkappa_{2} \in J$, such that $\varkappa_{2}<\varkappa_{1}$. Then, we have: 


$$
\begin{aligned}
& \left.\left.\mid \mathcal{Q} u\left(\varkappa_{2}\right)\right)\left[\vartheta\left(\varkappa_{2}\right)-\vartheta(a)\right]^{1-\gamma}-\mathcal{Q} u\left(\varkappa_{1}\right)\right)\left[\vartheta\left(\varkappa_{1}\right)-\vartheta(a)\right]^{1-\gamma} \mid \\
\leq & \frac{1}{\Gamma(\varrho)} \int_{a}^{\varkappa_{1}}\left(\left[\vartheta\left(\varkappa_{2}\right)-\vartheta(a)\right]^{1-\gamma} \vartheta^{\prime}(s)\left(\vartheta\left(\varkappa_{2}\right)-\vartheta(s)\right)^{\varrho-1}\right. \\
& {\left.\left[\vartheta\left(\varkappa_{1}\right)-\vartheta(a)\right]^{1-\gamma} \vartheta^{\prime}(s)\left(\vartheta\left(\varkappa_{1}\right)-\vartheta(s)\right)^{\varrho-1}\right)\left|\mathcal{H}_{u}(s)\right| d s } \\
& +\frac{1}{\Gamma(\varrho)} \int_{\varkappa_{1}}^{\varkappa_{2}}\left[\vartheta\left(\varkappa_{2}\right)-\vartheta(a)\right]^{1-\gamma} \vartheta^{\prime}(s)\left(\vartheta\left(\varkappa_{2}\right)-\vartheta(s)\right)^{\varrho-1}\left|\mathcal{H}_{u}(s)\right| d s \\
& +\left(\left[\vartheta\left(\varkappa_{2}\right)-\vartheta(a)\right]^{1-\gamma}\left|\mu\left(\varkappa_{2}\right)\right|-\left[\vartheta\left(\varkappa_{1}\right)-\vartheta(a)\right]^{1-\gamma}\left|\mu\left(\varkappa_{1}\right)\right|\right) \\
& b_{1} \frac{1}{\Gamma(\varrho)} \int_{a}^{b} \vartheta^{\prime}(s)(\vartheta(b)-\vartheta(s))^{\varrho-1}\left|\mathcal{H}_{u}(s)\right| d s \\
+ & \left(\left[\vartheta\left(\varkappa_{2}\right)-\vartheta(a)\right]^{1-\gamma}\left|v\left(\varkappa_{2}\right)\right|-\left[\vartheta\left(\varkappa_{1}\right)-\vartheta(a)\right]^{1-\gamma}\left|v\left(\varkappa_{1}\right)\right|\right) \\
& \left(\frac{a_{2}}{\Gamma(\varrho-\sigma)} \int_{a}^{\eta} \vartheta^{\prime}(s)(\vartheta(\eta)-\vartheta(s))^{\varrho-\sigma-1}\left|\mathcal{H}_{u}(s)\right| d s\right. \\
& \left.\frac{a_{2}}{\Gamma(\varrho-\sigma)} \int_{a}^{b} \vartheta^{\prime}(s)(\vartheta(b)-\vartheta(s))^{\varrho-\sigma-1}\left|\mathcal{H}_{u}(s)\right| d s\right) \\
& \left|\Delta_{1}\right|\left(\left[\vartheta\left(\varkappa_{2}\right)-\vartheta(a)\right]^{1-\gamma}-\left[\vartheta\left(\varkappa_{1}\right)-\vartheta(a)\right]^{1-\gamma}\right) \\
+ & \left|\Delta_{2}\right|\left(\left[\vartheta\left(\varkappa_{2}\right)-\vartheta(a)\right]^{1-\gamma}-\left[\vartheta\left(\varkappa_{1}\right)-\vartheta(a)\right]^{1-\gamma}\right) \\
0 & \text { as } \varkappa_{2} \rightarrow \varkappa_{1} .
\end{aligned}
$$

That implies:

$$
\left\|\mathcal{Q} u\left(\varkappa_{2}\right)-\mathcal{Q}\left(\varkappa_{1}\right)\right\|_{\mathbb{C}_{1-\gamma}^{\vartheta}} \rightarrow 0 \quad \text { as } \varkappa_{2} \rightarrow \varkappa_{1}
$$

is uniformly for all $u \in \mathbb{X}$. This means that $\mathcal{Q}$ is equicontinuous on $\mathbb{C}_{1-\gamma}^{\vartheta}(J)$. In consequence, $\mathcal{Q}$ will be relatively compact. As a result of the Arzelà-Ascoli theorem, we deduce that $\mathcal{Q}$ is completely continuous. Thus, $\mathcal{Q}$ is compact on $\mathbb{X}$.

Step-III: In this step, we need to show that the condition (s3) of Theorem 1 holds. Let for $v \in \mathbb{X}$, such that:

$$
u=\mathcal{P} u \mathcal{Q} v+\mathcal{S} u
$$

Then, we have:

$$
\begin{aligned}
& \left|[\vartheta(\varkappa)-\vartheta(a)]^{1-\gamma} u(\varkappa)\right| \\
& =\left|[\vartheta(\varkappa)-\vartheta(a)]^{1-\gamma}(\mathcal{P} u \mathcal{Q} v)(\varkappa)+[\vartheta(\varkappa)-\vartheta(a)]^{1-\gamma} \mathcal{S} u(\varkappa)\right| \\
& \leq[\vartheta(\varkappa)-\vartheta(a)]^{1-\gamma}|\mathcal{P} u(\varkappa)||\mathcal{Q} v(\varkappa)|+[\vartheta(\varkappa)-\vartheta(a)]^{1-\gamma}|\mathcal{S} u(\varkappa)| \\
& \leq[|\mathcal{G}(\varkappa, u(\varkappa))-\mathcal{G}(\varkappa, 0)|+|\mathcal{G}(\varkappa, 0)|] \\
& \times\left[[\vartheta(\varkappa)-\vartheta(a)]^{1-\gamma} \frac{1}{\Gamma(\varrho)} \int_{a}^{\varkappa} \vartheta^{\prime}(s)(\vartheta(\varkappa)-\vartheta(s))^{\varrho-1}\left|\mathcal{G}_{u}(s)\right| d s\right. \\
& +[\vartheta(\varkappa)-\vartheta(a)]^{1-\gamma}|\mu(\varkappa)| b_{1} \frac{1}{\Gamma(\varrho)} \int_{a}^{b} \vartheta^{\prime}(s)(\vartheta(b)-\vartheta(s))^{\varrho-1}\left|\mathcal{G}_{u}(s)\right| d s \\
& +[\vartheta(\varkappa)-\vartheta(a)]^{1-\gamma}|v(\varkappa)|\left(\frac{a_{2}}{\Gamma(\varrho-\sigma)} \int_{a}^{\eta} \vartheta^{\prime}(s)(\vartheta(\eta)-\vartheta(s))^{\varrho-\sigma-1}\left|\mathcal{G}_{u}(s)\right| d s\right. \\
& \left.+\frac{a_{2}}{\Gamma(\varrho-\sigma)} \int_{a}^{b} \vartheta^{\prime}(s)(\vartheta(b)-\vartheta(s))^{\varrho-\sigma-1}\left|\mathcal{G}_{u}(s)\right| d s\right)
\end{aligned}
$$




$$
\begin{aligned}
& \left.+[\vartheta(\varkappa)-\vartheta(a)]^{1-\gamma}\left|\Delta_{1}\right|[\vartheta(\varkappa)-\vartheta(a)]^{\gamma-1}+[\vartheta(\varkappa)-\vartheta(a)]^{1-\gamma}\left|\Delta_{2}\right|[\vartheta(\varkappa)-\vartheta(a)]^{\gamma-2}\right] \\
& +[\vartheta(\varkappa)-\vartheta(a)]^{1-\gamma}[|\mathcal{F}(\varkappa, u(\varkappa))-\mathcal{F}(\varkappa, 0)|+|\mathcal{F}(\varkappa, 0)|] \\
& \leq(\mathcal{A} \Phi+\Delta)[|\mathcal{G}(\varkappa, u(\varkappa))-\mathcal{G}(\varkappa, 0)|+|\mathcal{G}(\varkappa, 0)|] \\
& +[\vartheta(\varkappa)-\vartheta(a)]^{1-\gamma}[|\mathcal{F}(\varkappa, u(\varkappa))-\mathcal{F}(\varkappa, 0)|+|\mathcal{F}(\varkappa, 0)|] \\
& \leq(\mathcal{A} \Phi+\Delta)\left[L_{\mathcal{P}}\|u\|+\mathcal{G}^{*} \mid\right]+\left[L_{\mathcal{S}}\|u\|+\mathcal{F}^{*} \mid\right] .
\end{aligned}
$$

Thus, we obtain:

$$
\|u\|_{\mathbb{C}_{1-\gamma}^{\theta}} \leq(\mathcal{A} \Phi+\Delta)\left[L_{\mathcal{P}}\|u\|_{\mathbb{C}_{1-\gamma}^{\theta}}+\mathcal{G}^{*} \mid\right]+\left[L_{\mathcal{S}}\|u\|_{\mathbb{C}_{1-\gamma}^{\theta}}+\mathcal{F}^{*} \mid\right],
$$

and hence:

$$
\|u\|_{\mathbb{C}_{1-\gamma}^{\vartheta}} \leq \frac{(\mathcal{A} \Phi+\Delta) \mathcal{G}^{*}+\mathcal{F}^{*}}{1-\left[(\mathcal{A} \Phi+\Delta) L_{\mathcal{P}}+L_{\mathcal{S}}\right]} \leq \rho .
$$

This means that the condition (s3) of Theorem 1 holds.

Step-IV: Finally, we have:

$$
\begin{aligned}
M_{\mathcal{Q}}=\|\mathcal{Q}(\mathbb{X})\|_{\mathbb{C}_{1-\gamma}^{\vartheta}} & =\sup \left\{\|\mathcal{Q} u\|_{\mathbb{C}_{1-\gamma}^{\vartheta}}: u \in \mathbb{X}\right\} \\
& \leq \mathcal{A} \Phi+\Delta
\end{aligned}
$$

From above estimate and by (31), we obtain:

$$
L_{\mathcal{P}} M_{\mathcal{Q}}+L_{\mathcal{S}} \leq L_{\mathcal{P}}(\mathcal{A} \Phi+\Delta)+L_{\mathcal{S}}<1 .
$$

Hence, the condition (s4) of Theorem 1 holds. So, the operators $\mathcal{P}, \mathcal{Q}$, and $\mathcal{S}$ satisfy all conditions in Theorem 1; hence, the equation $\mathcal{P} u \mathcal{Q} u+\mathcal{S} u=u$ has a solution in $\mathbb{X}$. Consequently, the problem (4) has a solution on $J$.

\section{An Example}

In this portion, we support our analytical results by demonstrating a pertinent example to indicate the applicability of obtained proofs.

Example 1. We consider the following hybrid implicit FBVP:

$$
\left\{\begin{array}{l}
\mathcal{D}_{a^{+}}^{Q, \beta ; \vartheta}\left[\frac{u(\varkappa)-\left(\frac{\varkappa^{2}}{10}\left(\frac{1}{2}(|u|)+e^{-\varkappa}\right)\right)}{\left(\frac{1}{100} e^{\varkappa}+\frac{2+|u|}{8 e^{2+\varkappa}(1+|u|)}\right)}\right]=\frac{\sqrt{2} \pi}{4(4 \pi-\varkappa)^{2}}(1+|u|+|v|), \\
a_{1} \mathcal{I}_{a^{+}}^{1-\gamma ; \vartheta}\left(\frac{u(a)-\mathcal{F}(a, u(a))}{\mathcal{G}(a, u(a))}\right)+b_{1}\left(\frac{u(b)-\mathcal{F}(b, u(b))}{\mathcal{G}(b, u(b))}\right)=c_{1}, \\
a_{2} \mathcal{D}_{a^{+}}^{\sigma, \beta ; \vartheta}\left(\frac{u(\eta)-\mathcal{F}(\eta, u(\eta))}{\mathcal{G}(\eta, u(\eta))}\right)+b_{2} \mathcal{D}_{a^{+}}^{\sigma, \beta ; \vartheta}\left(\frac{u(b)-\mathcal{F}(b, u(b))}{\mathcal{G}(b, u(b))}\right)=c_{2}, \quad \eta \in(0,1) .
\end{array}\right.
$$

Let us consider the hybrid system (37) with specific data:

$$
\begin{gathered}
\varrho=\frac{3}{2}, \quad \beta=\frac{1}{4}, \gamma=\frac{13}{8}, \quad \sigma=\frac{2}{3}, \eta=\frac{3}{5}, a=0, \quad b=1, \\
a_{1}=a_{2}=b_{1}=b_{2}=1, c_{1}=\frac{1}{2}, c_{2}=\frac{3}{4}, \vartheta(\varkappa)=e^{\varkappa}, J=[0,1] .
\end{gathered}
$$


Our analysis of the given data shows that:

$$
\begin{gathered}
\mathcal{F}^{*}=\frac{1}{10}, \quad \mathcal{G}^{*}=\frac{1}{100}, \\
|\mathcal{F}(\varkappa, u)-\mathcal{F}(\varkappa, \bar{u})| \leq \frac{1}{10}|u-\bar{u}|, \\
|\mathcal{G}(\varkappa, u)-\mathcal{G}(\varkappa, \bar{u})| \leq \frac{1}{8 e^{2}}|u-\bar{u}|, \\
\mu(\varkappa)=\frac{\left[e^{\varkappa}-1\right]^{\gamma-1}}{\Lambda \Gamma(\gamma)} \omega_{3}-\frac{\left[e^{\varkappa}-1\right]^{\gamma-2}}{\Lambda \Gamma(\gamma-1)} \omega_{4},
\end{gathered}
$$

and

$$
v(\varkappa)=\frac{\left[e^{\varkappa}-1\right]^{\gamma-1}}{\Lambda \Gamma(\gamma)} \omega_{1}-\frac{\left[e^{\varkappa}-1\right]^{\gamma-2}}{\Lambda \Gamma(\gamma-1)} \omega_{2} .
$$

Hence, the hypotheses $(\mathbb{H} \mathbb{Y} \mathbb{P} 1)$ is satisfied with:

$$
L_{\mathcal{S}}=\frac{1}{10}, L_{\mathcal{G}}=\frac{1}{8 e^{2}}, L_{\mathcal{P}}=\left[e^{\varkappa}-1\right]^{1-\frac{13}{8}} \frac{1}{8 e^{2}} .
$$

Let $u, v \in \mathbb{R}$. Then, we have:

$$
|\mathcal{H}(\varkappa, u, v)| \leq \frac{\sqrt{2} \pi}{4(4 \pi-\varkappa)^{2}}(1+|u|+|v|),
$$

and so, the hypotheses $(\mathbb{H} \mathbb{Y} 1)$ and $(\mathbb{H} \mathbb{Y} \mathbb{P} 3)$ are satisfied with

$$
p(\varkappa)=q(\varkappa)=r(\varkappa)=\frac{\sqrt{2} \pi}{4(4 \pi-\varkappa)^{2}} .
$$

So:

$$
p^{*}=q^{*}=r^{*}=\frac{\sqrt{2}}{64 \pi} .
$$

By the given data, we get $\Lambda=0.1 \neq 0, L_{\mathcal{P}} M_{\mathcal{Q}}+L_{\mathcal{S}}=0.58<1$. Thus, all the conditions of Theorem 2 are satisfied. Hence, our problem (4) has at least one solution in $\mathbb{C}_{1-\frac{13}{8}}^{e^{\varkappa}}[0,1]$.

\section{Conclusions}

Nowadays, we need to develop and refine our capabilities, in order to generalize some recent results directly related to the subject of FDEs, for the purpose of expanding new trends in the area of these equations and their real-world applications.

Many authors have generalized some of the various fractional operators, utilizing traditional operators in fractional calculus. One of these operators is the generalized Hilfer fractional derivative, introduced by Sousa and Oliveira [12]. In this paper, we have successfully investigated the existence of solution for the $\vartheta$-Hilfer type nonlinear implicit hybrid FDE. The main result was obtained by applying Dhage's hybrid fixed point theorem for three operators in a Banach algebra [26-28]. The main results are illustrated with a numerical example. Further, our current problem was a general extension of the previous standard cases of implicit hybrid FDEs by assigning different values for all existing orders and parameters or defining the $\vartheta$ function in the aforesaid problem (4). The supposed problem (4), with given mixed integro-derivative boundary conditions, can describe some mathematical models of real and physical processes, in which some parameters are frequently acclimated to appropriate circumstances. So, the value of these parameters can change the impacts of fractional integrals and derivatives. 
Author Contributions: Conceptualization, A.B., H.A. and M.S.A.; formal analysis, A.B., M.S.A. and M.A.A.; methodology, M.S.A., H.A. and M.A.A.; resources, A.I. All authors have read and agreed to the revised version of the manuscript.

Funding: The authors received financial support from Taif University Researchers Supporting Project number (TURSP-2020/223), Taif University, Taif, Saudi Arabia.

Institutional Review Board Statement: Not applicable.

Informed Consent Statement: Not applicable.

Data Availability Statement: Not applicable.

Acknowledgments: The research was supported by the Taif University Researches Supporting Project number (TURSP-2020/223), Taif University, Taif, Saudi Arabia.

Conflicts of Interest: The authors declare no conflict of interest.

\section{References}

1. Kilbas, A.A.; Srivastava, H.M.; Trujillo, J.J. Theory and Applications of Fractional Differential Equations, North-Holland Mathematics Studies, 204; Elsevier Science B.V.: Amsterdam, The Netherlands, 2006.

2. Podlubny, I. Fractional Differential Equations; Academic Press: New York, NY, USA, 1999.

3. Samko, S.G.; Kilbas, A.A.; Marichev, O.I. Fractional Integrals and Derivatives, Theory and Applications; Gordon and Breach: Yverdon, Switzerland, 1993.

4. Hilfer, R. Applications of Fractional Calculus in Physics; World Scientific: Singapore, 2000.

5. Baskonus, H.M.; Sánchez-Ruiz, L.M.; Ciancio, A. New Challenges Arising in Engineering Problems with Fractional and Integer Order. Fractal Fract. 2021, 5, 35. [CrossRef]

6. Fernandez, A.; Ozarslan, M.A.; Baleanu, D. On fractional calculus with general analytic kernels. Appl. Math. Comput. 2019, 354, 248-265. [CrossRef]

7. Erdelyi, A. An integral equation involving Legendre functions. J. Soc. Indust. Appl. Math. 1964, 12, 15-30. [CrossRef]

8. Katugampola, U.N. New approach to a generalized fractional integral, Applied Mathematics and Computation. Appl. Math. Comput. 2011, 218, 860-865.

9. Diaz, J.B.; Osler, T.J. Differences of fractional order. Math. Comput. 1974, 28, 185-202. [CrossRef]

10. Oldham, K.B.; Spanier, J. The Fractional Calculus; Academic Press: New York, NY, USA; London, UK, 1974.

11. Almeida, R. A Caputo fractional derivative of a function with respect to another function. Commun. Nonlinear Sci. Numer. Simul. 2017, 44, 460-481. [CrossRef]

12. Sousa, J.V.D.C.; de Oliveira, E.C. On the $\psi$-Hilfer fractional derivative. Commun. Nonlinear Sci. Numer. Simul. $2018,60,72-91$. [CrossRef]

13. Jarad, F.; Abdeljawad, T. Generalized fractional derivatives and Laplace transform. Discret. Contin. Dyn. Syst. Ser. S 2020, 13, 709. [CrossRef]

14. Almalahi, M.A.; Abdo, M.S.; Panchal, S.K. Existence and Ulam-Hyers-Mittag-Leffler stability results of $\psi$-Hilfer nonlocal Cauchy problem. Rend. Del Circ. Mat. Palermo Ser. 2021, 70, 57-77. [CrossRef]

15. Almalahi, M.A.; Panchal, S.K.; Jarad, F. Stability results of positive solutions for a system of $\psi$-Hilfer fractional differential equations. Chaos Solitons Fractals 2021, 147, 110931. [CrossRef]

16. Almalahi, M.A.; Abdo, M.S.; Panchal, S.K. Existence and Ulam-Hyers stability results of a coupled system of $\psi$-Hilfer sequential fractional differential equations. Results Appl. Math. 2021, 10, 100142. [CrossRef]

17. Abdo, M.S.; Thabet, S.T.; Ahmad, B. The existence and Ulam-Hyers stability results for $\psi$-Hilfer fractional integrodifferential equations. J. Pseudo-Differ. Oper. Appl. 2020, 11, 1757-1780. [CrossRef]

18. Almalahi, M.A.; Panchal, S.K.; Jarad, F.; Abdeljawad, T. Ulam-Hyers-Mittag-Leffler stability for tripled system of weighted fractional operator with TIME delay. Adv. Differ. Equ. 2021, 1, 1-18. [CrossRef]

19. Abdellatif, B.; Sina, E.; Azhar, H.; Shahram, R. The generalized U-H and U-H stability and existence analysis of a coupled hybrid system of integro-differential IVPs involving $\vartheta$-Caputo fractional operators. Adv. Differ. Equ. 2021, 95, 1-21. [CrossRef]

20. Shatanawi, W.; Boutiara, A.; Abdo, M.S.; Jeelani, M.B.; Abodayeh, K. Nonlocal and multiple-point fractional boundary value problem in the frame of a generalized Hilfer derivative. Adv. Differ. Equ. 2021, 294, 1-19. [CrossRef]

21. Abdo, M.S.; Panchal, S.K.; Saeed, A.M. Fractional boundary value problem with $\vartheta$-Caputo fractional derivative. Proc. Indian Acad. Sci. Math. Sci. 2019, 129, 65. [CrossRef]

22. Wang, J.R.; Zhang, Y.R. Nonlocal initial value problems for differential equations with Hilfer fractional derivative. Appl. Math. Comput. 2015, 266, 850-859. [CrossRef]

23. Derbazi, C.; Hammouche, H.; Benchohra, M.; Zhou, Y. Fractional hybrid differential equations with three-point boundary hybrid conditions. Adv. Differ. Equ. 2019, 2019, 125. [CrossRef]

24. Salim, A.; Benchohra, M.; Lazreg, J.E.; Nieto, J.J.; Zhou, Y. Nonlocal Initial Value Problem for Hybrid Generalized Hilfer-type Fractional Implicit Differential Equations. Nonautonomous Dyn. Syst. 2021, 8, 87-100. [CrossRef] 
25. Srivastava, H.; Tomovski, Z. Fractional calculus with an integral operator containing a generalized Mittag-Leffler function in the kernel. Appl. Math. Comput. 2009, 211, 198-210.

26. Dhage, B.C. A fixed point theorem in Banach algebras involving three operators with applications. Kyungpook Math. J. 2004, 44, $145-155$.

27. Dhage, B.C. A nonlinear alternative in Banach algebras with applications to functional differential equations. Nonlinear Funct. Anal. Appl. 2004, 8, 563-575.

28. Dhage, B.C. Basic results in the theory of hybrid differential equations with mixed perturbation of second type. Funct. Diff. Equ. 2012, 19, 87-106. 